\title{
Act Collectively: Opportunities for Technologies to Support Low-Income Children with Asthma
}

\author{
Hee Young Jeong ${ }^{1}$, Gillian R. Hayes ${ }^{2}$, Tae-Jung Yun ${ }^{1}$, Ja-Young Sung ${ }^{1}$, Gregory D. Abowd ${ }^{1}$, and Rosa I. Arriaga ${ }^{1}$ \\ ${ }^{1}$ School of Interactive Computing \\ Georgia Institute of Technology \\ $855^{\text {th }}$ Street NW, Atlanta, GA 30332, USA \\ [hyj, tjyun, jsung, abowd]@gatech.edu, \\ arriaga@cc.gatech.edu \\ ${ }^{2}$ Department of Informatics \\ Donald Bren School of Information and Computer \\ Science, University of California, Irvine \\ Donald Bren Hall 5042, Irvine, CS 92697-3440, USA \\ gillianm@ics.uci.edu
}

\begin{abstract}
Asthma is one of the most common childhood chronic diseases in the world, and the nature of paediatric asthma management inherently requires intense collaborative care. In this paper, we report the results of a multi-method qualitative inquiry aimed at understanding paediatric asthma management and the potential for technologies to support low-income families. Our findings highlight three themes around the challenges to managing paediatric asthma faced by low-income families in the US: information sharing about environmental triggers, health communication and coordination, and the need for social support. Additionally, we critique existing solutions in light of the needs of families and suggest new avenues for designing for effective asthma management.
\end{abstract}

Healthcare, Communication, Coordination, Low-income children with asthma, Collaborative technologies

\section{INTRODUCTION}

Asthma is characterized by coughing, wheezing, difficulty with breathing and is a lifelong condition that typically begins in childhood. It is estimated that around 300 million people in the world have asthma (Masoli et al. 2004). It is one of the most common childhood chronic illnesses worldwide (Masoli et al. 2004). In particular, the prevalence of asthma in Wales is the highest; Ireland is ranked as the third highest; and the US as the fifth highest (Masoli et al. 2004). We use low-income families in the US as a case study of how asthma affects lowincome families and how they deal with the challenged presented by paediatric asthma.

Family members with paediatric chronic illnesses are burdened with time spent in transit or at physician's offices and hospitals rather than at work, school or home (Szefler and Leung 2001). Paediatric asthma management requires financial resources, education, and high quality communication with healthcare providers (Ortega and Caldron 2000). However, low-income families may not have access to these resources. Additionally, low-income households tend to have low health literacy even if they are otherwise literate (Williams et al. 1998). Thus, many researchers have argued that management strategies for paediatric asthma should focus on high-risk populations, such as low-income children (Lara et al. 2002), who lack a regular source of care (Sondik et al. 2009).

To understand the needs of these families with limited resources, we conducted our study in the US and focused on families who use Medicaid, the government subsidized healthcare. Although this study focused on the US healthcare context, our paper aims to draw the design implications for an international context. Thus, we set out to answer three research questions:

1) What are the challenges experienced by collaborative asthma care teams?

2) What strategies do caregivers currently use to address these challenges collectively?

3) How can we support current strategies and develop new ones through the use of technology?

In this paper, we report findings from a series of qualitative empirical studies designed to address these questions. We first present three primary themes underpinning the challenges of paediatric asthma care for low-income families. These themes are the sharing of information around environmental triggers, strategies for improving 
healthcare communication and coordination, and the need for social support. Once these themes are identified, we reflect on related research and our findings to uncover opportunities for future design solutions specifically related to these issues.

\section{METHODS}

The ecosystem surrounding paediatric asthma care includes a wide variety of stakeholders, triggers, symptoms, and treatments. To triangulate the needs of families, children, medical health professionals and other relevant stakeholders, we tailored our approach to each stakeholder group and context as part of a multi-method qualitative approach (Mackay and Fayard 1997). We first conducted exploratory fieldwork to further define our scope of inquiry and develop our interview guides. We conducted observations at asthma related events and took detailed field notes. Following this work, we conducted interviews with seven low-income parents with asthmatic children between the ages of seven and thirteen in their homes, all of whom had an asthma diagnosis and used Medicaid services. We interviewed six providers (a paediatric pulmonologist, an allergist, a nurse, a respiratory therapist, a physician assistant, and a certified asthma educator who was a school nurse) in their offices. We recruited participants through two local asthma clinics and Craigslist. Each home interview lasted approximately 90 minutes and included a home tour, an interview with the primary caregivers (parents and one grandparent), and an interview with the children without parents when possible $(n=6)$. The interviews were semi-structured, allowing participants to drive the discussion towards those issues most important to them, but with a focus on asthma management and collaborative care. All interviews were audio-recorded and transcribed. We also conducted a 90-minute group interview with four CHWs, two of whom had been shadowed previously.

We employed an inductive qualitative analysis approach (similar to thematic or affinity analysis) to identify emergent themes pertaining to the design of technological solutions to address the needs of low-income children with asthma, their families, and professional caregivers. To achieve this, three researchers independently coded the raw transcripts by capturing interesting events. Then, the researchers worked together closely to find causal and contextual relationships between the phenomena. All transcripts and field notes were coded using Atlas.ti. Through group discussion, we then categorized these findings into three major themes: sharing of environmental trigger information, communications and coordination, and social support. Our findings also lead to design guidelines and opportunities.

\section{FINDINGS}

Management of paediatric asthma care, particularly for low-income families, is a highly collaborative endeavour. In this section, we describe the current barriers to care, strategies, and design opportunities to mitigate or overcome these challenges.

Before describing the issues specific to asthma care, however, it is important to note three issues that may arise in designing technology for lowincome populations more generally. First, designers must consider access to technology and the growing "digital divide" by supporting sociocultural and financial barriers. For example, leveraging existing infrastructures such as computers in public libraries and schools for Internet access could be useful in supporting health communication. Second, the technologies should be flexible and customizable to support the needs, capabilities, and technology access for a diverse set of stakeholders in various contexts. Third, nontraditional platforms should be considered for the development of solutions for low-income families. For example, low-income families in the US tend to have greater access to mobile phones than other Internet technologies (Lenhart, 2010). However, it is possible that accessibility to mobile phones would vary in other countries. In the remainder of this section, we describe our findings focused on sharing of environmental trigger information, communicating among a complex and diverse caregiver network, and the need for social support for both caregivers and children with asthma.

\subsection{Sharing Environmental Trigger Information}

Indoor and outdoor environmental trigger management is one of the primary strategies for reducing asthma symptoms. However, it can be particularly challenging for families who reside in low-income rental housing,

\begin{abstract}
"Some families live in an environment where they are exposed to [a] tremendous number of triggers. They have a leaky roof... problems with their pets.... poverty is a tremendous trigger for asthma." (Respiratory therapist)
\end{abstract}

To manage environmental triggers at home, families in our sample reported using either broadcast media or CHW services. In particular, most families described using television or radio to check allergen levels, weather and temperature. 
"We watch TV... I try to make sure I turn on the news every morning, so I can keep up with the smog alert if it's moderate, severe... and all of that stuff. I try to stay on top of all of that... If it's not a high pollen count, then it won't show it, and we can let him go outside." (Married parents of seven-year-old boy with asthma)

However, this broadcast model can provide ambiguous information, and as noted in the example above, parents may make inferences based on missing data (no pollen count on the news). The parent above notes that they assume that the pollen count must be low enough to let the child go outdoors. Furthermore, these channels can mean that low-income families do not supply information about the full range of outdoor as well as indoor environmental triggers (e.g., Indoor: tobacco smoke, dust mites, cockroaches; Outdoor: lead, particulate matter) nor the kind of localized information needed by families. Thus, new technologies must be designed and developed to support sharing of local environmental information in ways that are accessible for low-income families.

As noted previously, low-income families tend to be more likely to have access to the Internet through mobile devices (Lenhart, 2010). Additionally, as more and more youth become accustomed to using mobile devices, both for low-income and other asthma patients, participatory sensing and data sharing solutions must be built for mobile devices.

In addition to broadcast media, many families in our sample described using $\mathrm{CHW}$ services to share information and manage their poor living environments. CHWs serve as intermediaries in communication among caregivers, often through phone calls and paper-based notes. Unknown environmental triggers can impact children with asthma indicating the need for cooperative action supported by local communities, currently undertaken largely by $\mathrm{CHWs}$ if at all. To support this kind of action on a broader scale and in environments in which CHWs do not work, collaborative technologies should be designed to encourage local communities to identify collectively what types of local environmental factors can be asthma triggers. These kinds of technologies for collective social action could also support families when they are away from home. Each local environment has its own environmental triggers, such as pollution near ports or pollen near forests. For instance, one family described a vacation trip:

"Before we went and stayed at the resort, I had to talk to management... had to request special mattresses and stuff like that. It's not like we can just jump up and go stay at a hotel."(Divorced mother of nine-year-old girl with asthma)
In this example, the family had the means to stay at a resort that could accommodate their needs. However, in similar discussions with other families, it became clear that this sort of solution would not be available for most of them. They noted that they typically travelled to lower priced hotels with fewer amenities or that they stayed with family and friends who had pets, smoke, or expose their child to other asthma triggers. Thus, increased sharing of information among families dealing with these same issues could improve the ability of families to make decisions about affordable travel plans that address their particular needs around asthma. These types of solutions could also support environmental experts encouraging social movements to improve local conditions.

In relation to this finding, potential design opportunities would be found in low-cost platforms that allow children and their caregivers to report symptoms and to link these symptoms to databases of environmental information, both that sense collected by central organizations (e.g., pollen counts) as well as and those reported by individuals (e.g., the appearance of mould in community housing). Participation in their own health can empower individuals and groups to improve their communities, changing the nature of health monitoring in low-income areas from one of surveillance to one of community action.

\subsection{Healthcare Communications and Coordination}

Communicating and coordination around medication adherence and other care-giving activities can be critical for chronic disease management, particularly for people who are not primarily responsible for their own health such as elders and children (Consolvo et al. 2004; Kientz et al. 2006). A wide variety of caregivers are involved in paediatric asthma management (Jeong and Arriaga 2009), particularly for low-income families. Caregivers communicate regularly with one another about the child's medication schedules, and symptoms, because asthma treatment strategies frequently change based on symptoms triggered by physical activities, emotions, and environmental conditions. This communication serves to ensure continuity of treatment and management across a variety of situations both in and out of the home. This kind of communication can be particularly important and difficult for low-income families who rely more on informal care than middle-class families (Beadle, 2006). Here, we describe challenges to communication, strategies for seeking $\mathrm{CHW}$ service, and inclusion of children as active participants in communication.

\subsubsection{Communication among Caregivers}


Children spend a large percentage of their lives in school. Thus, parents we interviewed were particularly concerned about sharing information with school staff.

"I would make sure I communicated with the coach. If he doesn't look right - if he looks like he's breathing too hard, sit him to the side."(Married mother of four boys with asthma)

Parents also often expressed wanting schools to seek permission before treating their children. For example,

"I have informed the schools that this is her condition .... if she does have a fever they can give her Tylenol but they need to call me before they administer it." (Divorced mother of sevenyear-old girl with asthma)

Despite the eagerness on the part of some parents to open these communication lines, others are less forthcoming with information needed by schools.

\begin{abstract}
"Most families... don't share the information with the school. They let the child carry the medicine on their person without the school's knowledge..." (Registered school nurse)
\end{abstract}

This reluctance to share was sometimes due to inherent disinterest on the part of the parents to share. It may also, however, be related to challenges in sharing information using current means. Parents described heavily relying on phone calls in communicating with school staff and medical professionals. However, phone calls are not always effective, because communicating often involves receiving calls from school staff, then calling medical professionals, waiting for return calls, and then returning the call to the school staff to verify appropriate treatment. This process at its best can result in delays and inaccuracy. However, in our data, the issue of keeping accurate functional phone numbers for a population with difficulty paying their phone bills emerged as a substantial challenge. In addition to parent-school communication, many stakeholders described wanting greater school-provider communication. For example, school staff may need to seek advice from medical professionals familiar with a particular child before treating the child due to the wide variety of medications any one child may be taking.

\subsubsection{Involving Children in Care Coordination}

The children themselves can also be involved in communication and coordination as they mature. For example, a grandmother describes communicating directly with the child about his medication:

"He comes over here in the morning when he goes to school. First, I say 'Did you take your medicine this morning?', 'I took it.' that's the first thing I ask...." (Grandmother of 13-year-old boy with asthma)

Children with asthma and their caregivers sometimes report different symptoms (Lara et al. 1998). Thus, incorporating children with asthma into their care can help provide a more complete picture. Empowering children in their own care, however, can be challenging for adults.
"I used to go to the school every day to make sure she was getting her puffs.... But the nurse was like, 'Well, mom, you've got to back off and give her independence, and let her try to understand that she's got to manage some of the parts of this situation herself... It's hard. I'm a mom." (Divorced mother of nine-year-old girl with asthma)

However, children can and do self-manage; they often reach a point at which they are the experts in their own care:
"She'll tell me, 'Mom, I need to start taking my Singulair again,' because it's somewhat seasonal... she knows when she needs to start taking her medication more often...." (Single mother of eight-year-old girl with asthma)

In some cases, mobile phones (typically pre-paid) are provided to children to use in emergencies. However, US schools where the children in this study were enrolled tend to have restrictions on the use of mobile devices (Cramer and Hayes 2010). The tension between providing access to communications technologies for certain purposes but not for others presents opportunities and challenges for the development of new applications for children with chronic health conditions.

Additionally, children, even those described as independent by their parents, may regard medication management as an caregiver's job.

"I feel like pressure up here around my chest, and I just sit down for a while... if I'm home I just tell my mom, and she'll probably give me...a treatment." (12 year old boy with asthma)

Thus, involvement of children in their own care, to the degree appropriate and preferred by both caregivers and patients, is an open challenge.

To design novel collaborative technologies, they should include children as active participants. Applications that can run on school or library computers are a promising option for children who do not have access to these technologies at home. Furthermore, given the recent trend for parents to provide low cost mobile phones to their children for emergency purposes, the same mobile applications that might be appropriate for low-income adults may be useful for their children. 


\subsection{Social Support}

Social support can be important in generating positive health outcomes for chronic illness. However, in paediatric asthma care, both caregivers and children currently experience deficits in the social support available to them. Social networking sites and online-health interventions can provide accessible communication systems for low-income populations (Ellison et al. 2007). In this section, we describe how both parents and children receive social support to help them in their treatments and in destigmatizing their experiences.

\subsubsection{Parental Social Support}

Despite the availability of social support, such as online communities, social networking sites, and public asthma education seminars, few families in our study described using these services. None of the caregivers we interviewed had registered with online support groups. In fact, none of them even reported knowing that these social networking services existed when explicitly prompted about them, indicating the need for education and advertisement.

In the offline world, social support can come from local support groups.

"I think [a local support group] would be great especially for our younger mothers, because they don't know the severity of asthma." (Married mother of four boys with asthma)

\begin{abstract}
"We had a team support group you know whether it's the grandparents or whatever... because it's different as a team... and always have a community health worker at that support group session to help them along with things." (CHW1)
\end{abstract}

However, it can be difficult for low-income families, already incredibly busy and with challenges in transportation and child care, to take time out to organize or even to just attend such a support group.

Families share the burden of the asthma experience with peers and friends rather than only with medical professionals through chatting, phone calls, or text messaging. Information shared through these support networks can positively impact asthma diagnosis and management.

"My friend was one of the people that told me that my boy had asthma when the doctors said he didn't, and she would tell me to look into it or maybe I need to change doctors...." (Single mother of 11-year-old boy with asthma)

These results indicate the need to develop innovative technological solutions for receiving social support and connecting with other people with whom they can share information easily and quickly.

\subsubsection{Social Support for Children with Asthma}

The children in this study and their parents all expressed their desire to have "normal" lives without limitations on activities that can create stress and social isolation.

\section{"He's never lived a nomal childhood... It's like he'll come in here and he'll sit on the couch and he just won't say anything... It kind of gets him down a little bit." (Married parents of seven-year- old boy with asthma)}

Our results indicate that these issues may be even more severe for boys than girls, who have greater pressures to act tough and play sports.

\begin{abstract}
"Some fathers as far as they're concerned they want to make the boys to man up... You may have asthma... That's just a little cough, get it, come on, cough it out! get going!" (CHW1)
\end{abstract}

This potential for a gender effect leaves open research questions about how strong these effects are and how one might design with considerations for gender.

The differences felt by children with asthma compared to their friends without it can lead children to feel burdened and frustrated about their symptoms. In response, some families described experiences in which children refused to use rescue inhalers, did not request help in school or began counselling to deal with depression and social isolation. To help normalize the condition for their children, parents who can afford it may send their children to specialized "asthma camps".

\begin{abstract}
"Sometimes my other friends have asthma, like my best friend... he told me he got asthma like at the age of 3 , and I told him I had mine since I was born. He was like it must be hard... They tell me they have asthma and you're like okay you must be more careful about what you're doing." (12-year-old boy with asthma)
\end{abstract}

Unfortunately, for many low-income children, these resources are financially out of reach, suggesting technological opportunities that can provide social support without children leaving their local areas.

There are a variety of potential technology design paths available for the delivery of online social support for children with asthma. As low-income children gain greater access to social networking technologies through mobile phones and community-based computers, these multi-purpose platforms may serve some of this need. Additionally, context-appropriate solutions should 
be developed for school and other places children spend large amounts of time. In addition to increased integration with peers who do not have asthma, social support from those with asthma can help to normalize the condition for impacted children. Development of online health communities or collaborative games designed specifically for asthma can meet some of this need. However, to address the limited access to technological resources for low-income families, mobile applications that can be used on low-cost prepaid mobile phones as well as other low-cost solutions should be developed.

\section{RELATED WORK AND DISCUSSION}

In this section, we describe previous research efforts related to our findings and design opportunities. We frame our discussion around these themes: street science and environmental information sharing, collaborative technologies for health, and social support for the underserved.

\subsection{Street Science and Environmental Information Sharing}

Environmental information sharing is closely related to street science (Corburn, 2005) and communitybased interventions. For example, the AirBeat project enhanced partnerships among community residents and groups, increasing awareness of asthma triggers in local communities (Loh et al. 2002). To monitor outdoor air quality, they used four communication venues (website, telephone hotline, project participants, and television). However, this research does not address the need to collect data about shared indoor spaces and other localized issues that emerged in our data.

In other work, mobile phones have been actively used in street science to connect community members to professional practices (e.g., local environment experts) (Corburn, 2005). These communication technologies can provide real-time community-based information about asthma triggers. For example, Alexander explored how a population can work together to identify common asthma risk areas by collecting data about the frequency of using metered-dose inhalers with GPS (Freifeld et al. 2010).

Although this related research is promising, these efforts have not addressed the socio-technical context of low-income families. In particular, these solutions often require access to expensive smartphones or other high-end portable devices that no interviewees reported owning. For instance, although all the caregivers who participated in our study have mobile phones, they typically described using low-cost phones and paying only for minimal plans with voice or potentially a short messaging service capability but not Internet. Thus, there are opportunities to explore collaborative sensing and information sharing for low-income families as we describe in the finding section.

\subsection{Collaborative Technologies for Healthcare}

Previous research on collaborative healthcare technologies for families has focused on a wide variety of populations, including diabetics, elders, pregnant women, and children with special needs. For example, interactive web sites were used to support asynchronous communication between families and physicians in paediatric intensive care unit patients (Braner et al. 2004). Additionally, teams of professional and family-based care providers cooperatively monitor health related activities and share the activity information in their daily routines with other relevant people through a variety of communication technologies (Consolvo et al. 2004). However, challenges still exist for providing high quality care in teams, particularly for those as diverse as the ones supporting lowincome children with asthma.

Although studies have shown that communications technologies can support cooperation among complex care teams, the multitude of people involved in caring for paediatric asthma complicates this communication. For example, these solutions have not yet been explored for use with large care teams that include children, family members, school staff, medical professionals, and CHWs. Besides, there has been limited exploration of the communication between school staff and clinicians, a substantial need that emerged in our data. Additionally, when health technologies are built to consider children, they typically target educational interventions rather than truly incorporating children into the communication and information sharing practices (Ngo-Metzger et al. 2010).

\subsection{Social Support for the Medically Underserved}

The medically underserved population experiences economic, cultural, and linguistic barriers in receiving healthcare services (HRSA, 2007). To overcome healthcare disparities, community and social support can be critical, and collaborative systems have great potential to enhance this support at both the individual and organizational level. For example, at the individual level, EatWell is a phone-based system for sharing individual experiences with nutrition information can facilitate healthcare communication in low-income communities (Grimes et al. 2008). At the organizational level, coordination technologies in 
private, nonprofits social service organizations can enhance cooperative care networks between healthcare providers and the homeless (Le Dantec and Edwards 2008). These efforts have explicitly focused on low-income patients and thus provide important groundwork for our study, which addresses a different domain problem, paediatric asthma.

Despite the positive outcomes shown in much of the related literature, our findings on social support indicate that these technologies may be inaccessible or unknown to low-income families. Both the literature and our findings demonstrate that community support is critical for medically underserved populations. However, existing collaborative technologies do not fully consider the context of low-income children with asthma who require intense collaborative care and wish to have lives more similar to healthy children.

\section{CONCLUSION}

Effective management of paediatric asthma is a significant health concern world wide, and it is both more prevalent and more challenging for lowincome families in the US because of the lack of continual medical access due to lack of health insurance. In this work, we conducted an empirical study of paediatric asthma management for this population. It reveals three themes related to the practices of diverse collaborative care teams: managing environmental triggers and sharing information with local communities, tracking and sharing asthma symptoms and treatment information across the caregiver network, and the need for and solutions to provide social support among people beyond local communities. Drawing from these results, we presented a variety of potential opportunities for designing new technologies to support diverse care teams and empower both children and their families in thinking locally and acting collectively about their environments, coordinating communication and care, and facilitating social support. While our studies and findings are grounded in the specific details of low-income paediatric asthma management in the US, the design opportunities discussed may also be suitable for other paediatric chronic care situations, both in the U.S. and elsewhere. Our recommendations can motivate and inform those interested in collaborative technologies addressing chronic health concerns.

\section{REFERENCES}

Beadle, M. (2006) Children in low-income families: summary of the urban institute and child trends roundtable on children in low-income families. The Urban Institute, Washington, DC, USA.

Braner, D.A. Lai, S. Hodo, R. Ibsen, L.A. Bratton, S.L. Hollemon, D. and Goldstein, B. (2004) Interactive web sites for families and physicians of pediatric intensive care unit patients: A preliminary report, Pediatr Crit Care Med., 5, 434-439.

Consolvo, S. Roessler, P. Shelton, B.E. LaMarca, A. Schilit, B. and Bly, S. (2004) Technology for care networks of elders, IEEE Pervasive Computing, 3, 22-29.

Corburn, J. (2005) Street science: community knowledge and environmental health justice. MIT Press, Cambridge, MA, USA.

Cramer, M.D. and Hayes, G.R. (2010) 'Acceptable use in the age of connected youth: how risks, policies, and promises of the future impact student access to mobile phones and social media in schools', IEEE Pervasive Computing, 17-20 May, p. 37-44.

Freifeld, C.C. Chunara, R. Mekaru, S.R. Chan, E.H. Kass-Hout, T. Iacucci, A.A. and Brownstein, J.S. (2010) Participatory epidemiology: use of mobile phones for community-based health reporting, PLoS Medicine, 7, e1000376.

Grimes, A., Bednar, M., Bolter, J.D., Grinter, R.E. (2008) EatWell: Sharing nutrition-related memories in a low-income community. In Proc. CSCW 08, San Diego, CA. 8-12 Nov., pp. 87-96, ACM Press, NY, USA.

HRSA (2007) Community health worker national workforce study, U.S. Department of Health and Human Services, Health Resources and Services Administration: Rockville, MD, USA.

Jeong, H. and Arriaga, R.I. (2009) Using an ecological framework to design mobile technologies for pediatric asthma management. In Proc. MobileHCl 09, Bonn, Germany, 15-18 Sep., ACM Press, NY, USA.

Kientz, J.A. Hayes, G.R. Abowd, G.D. and Grinter, R.E. (2006) From the war room to the living room: decision support for home-based therapy teams. In Proc. CSCW 06, Banff, Alberta, Canada, 4-8 Nov., pp. 209-218, ACM Press, NY, USA.

Lara, M. Duan, N. Sherbourne, C. Lewis, M.A. Landon, C. Halfon, N. and Brook, R.H. (1998) Differences between child and parent reports of symptoms, Pediatrics, 102, e68. 
Lara, M., et al. (2002) Improving childhood asthma outcomes in the united states: a blueprint for policy action, Pediatrics, 109, 919-930.

Le Dantec C.A. and Edwards, W.K. (2008) The view from the trenches: organization, power, and technology at two nonprofit homeless outreach centers. In Proc. CSCW 08, San Diego, CA. 8-12 Nov. pp. 87-96, ACM Press, NY, USA.

Lenhart, A. (2010) Cell phones and american adults: they make just as many calls, but text less often than teens, Pew Research Center, http://pewinternet.org. (17 Jan. 2011).

Loh, P. Sugerman-Brozan, J. Wiggins, S. Noiles, D. and Archibald, C. (2002) From asthma to AirBeat: community-driven monitoring of fine particles and carbon in Roxbury, MA. Environ Health Perspect, 110, 297-301.

Mackay, W. and Fayard, A. (1997) HCl, natural science and design: a framework for triangulation across disciplines. In Proc. DIS 97, Amsterdam, 1820 August, pp. 223-234. ACM Press, NY. USA.

Masoli, M. Fabian, D. Holt, S. and Beasley, R. (2004) The global burden of asthma: executive summary of the GINA dissemination committee report. Allergy, 59, 469-478.

Ngo-Metzger, Q. Hayes, G.R. Chen, Y. Cygan, R. and Garfield, C.F. (2010) Improving communication between patients and providers using health information technology and other quality improvement strategies, Med Care Res and Rev, 246S-267S.

Ortega, A.N. and Caldron, J.G. (2000) Pediatric asthma among minority populations, Current Opinion in Pediatr., 12, 579-583.

Sondik, E.J. Madans, J.H. and Gentleman, J.F. (2009) Summary health statistics for U.S. children: National Health Interview Survey 2008, 10, 224.

Swider, S.M. (2002) Outcome effectiveness of community health workers: an integrative literature review, Public Health Nurs., 19, 11-20.

Szefler, S.J. and Leung, D.Y.M. (2001) Severe asthma: pathogenesis and clinical management. Informa Healthc are, NY. USA.

Williams, M.V. Baker, D.W. Honig, E.G. Lee, T.M. and Nowlan, A. (1998) Inadequate literacy is a barrier to asthma knowledge and self-care, Chest, 114, 1008-1015. 